a tool of intercommunication became available with the publication in 1951 of the "Interlingua-English Dictionary" and the "Interlingua Grammar" (Storm Publishers, 80 East Eleventh Street, New York 3). This was the culmination of twenty-seven years of research by the International Auxiliary Language Association in New York. Interlingua was developed by taking the words of vocabularies of the European languages (including English), standardizing or rationalizing them to remove regional irregularities and peculiarities, and constructing a simple grammar for use with the resultant vocabulary. An international language was obtained, which in a large measure is already in use and which can be read practically at sight by every educated person. The basic criterion for the acceptance of a word for the Interlingua vocabulary is that it must be present in corresponding forms and with corresponding meanings in at least three of the four language units: (1) Italian, (2) Spanish, and/or Portuguese, (3) French, and (4) English-with German and Russian as possible substitutes.

Impressed by the easy readability of Interlingua, Cleveland began, in May 1952, the publication in this language of a small periodical, Spectroscopia Molecular. It contains brief reports of new research results, summaries of important articles in the current literature, reports of spectroscopic meetings, reviews of now books, a calendar of future spectroscopic events, details about new instruments, and news about spectroscopists and recent developments. The experience of this small periodical provides a proof of the utility of Interlingua for intercommunication in the world of science.

Perhaps the greatest use of Interlingua to date has been in medicine. More than twenty medical journals publish extracts in Interlingua with all their original articles. In addition, Interlingua has been used for summaries in the programme of seven international medical congresses. Science News Letter publishes each month a summary in Interlingua of recent scientific developments.

To assist editors and authors who wish to study the possibility of increasing the extension of their work by the use of Interlingua, Science Service, Inc., has established an Interlingua Division. This is located at 80 East Eleventh Streot, Now York 3, and is under the direction of Dr. Alexander Gode.

\title{
RESEARCH AND DEVELOPMENT
}

$\mathrm{C}$

AN the effectiveness of research and development be measured ? More precisely-given a certain amount of research effort, in a given field, can the rate at which useful results will emerge be predicted ? These are some of the questions asked during a recent intensive study, "Basic Research in the Navy," carried out for the Naval Research Advisory Committee by Arthur D. Little, Inc. (Industrial Bulletin of Arthur D. Little, Inc., 369, Nov, 1959). In its report to the Secretary of the Navy, the Committee recommended further "research on research"; one approach is through a mathematical model describing the mechanisms of discovery and application.

The model describes a two-stage process; in the first stage, knowledge is discovered, and brought from the realm of the unknown into the known. In the second stage, combinations of the known facts are fitted together and applied. Formulation of the inventive process in this way emphasizes one important point : for every invention there is a key fact, or relation, or principle - the last to be discovered of all those necessary before the invention could be made. From this it follows that the date of discovery of this key fact is the earliest date upon which the invention could be made at all-although, of course, many inventions are not made until long after the discovery of the key fact.

There is a body of available knowledge, containing a certain number of key facts, corresponding to an equal number of possible inventions. If no new knowledge is added by research, these represent all the inventions which can be made. But the more research effort is applied, the more new facts come to light. It is also proportional to the size of the pool of (undiscovered) knowledge. The consequences of this ratio are interesting; the equation describing the rate of depletion of basic knowledge-the unknown subject-is an exponential decay curve like that used to describe the rate of decay of a radioactive substance. This is the model for the first stage of the research process.
The second stage concerns the application of discovered facts through invention. Here again the rate of progress is proportional to the amount of effort expended and to the size of the pool of know. ledge-in this case, the number of discovered, but unapplied, facts. Yet a fact may be applied many times, in different combinations with other facts. It is not the unit of knowledge that is importantrather, it is the conclusions drawn from a number of units. To determine a rate for the second stage properly, one must invoke the techniques of modern algebra.

There is, also, the question of the time-lag between discovery and application; for the second stage, as well as the first, one can draw an analogy with the process of radioactive decay. The body of known but unapplied facts is considered, on this model, to have a well-defined half-life, but the expected time for any one fact to be applied may range from very short to extremely long. The expected half-life itself is a variable quantity which depends on the amount of effort expended on development work.

By putting these various factors together, for the second stage an $\mathrm{S}$-shaped curve is obtained indicating first a slow rate of application, then a more rapid increase, and finally a plateau; curves of this type are familiar to economists for describing the development of a market or a new product. This is the model for the inventive process, and it is confirmed by observation.

Perhaps the most interesting feature of the model is that an optimum ratio for the effort to be applied to the two stages of discovery and application of knowledge can be derived. That is, one can in theory answer the question: For maximum productivity of a research programme, what percentage of the budget should be devoted to basic research, and what part to applied research? The model suggests that to develop one large and complex field, basie research should not fall below about 20 per cent of the total research budget. 